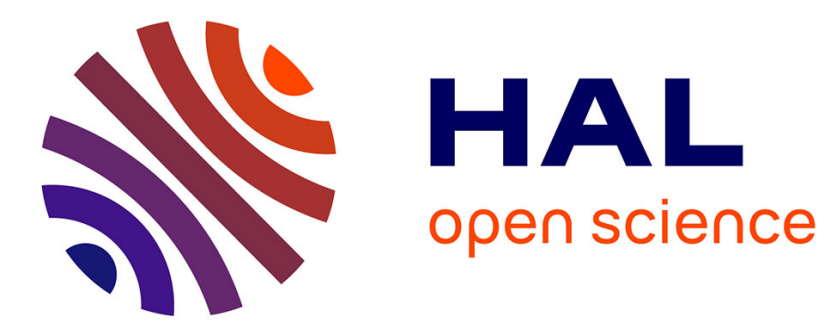

\title{
New Binary Single Side Band CPM
}

\author{
Haïfa Farès, Christian D.C. Glattli, Yves Louët, Christophe Moy, Jacques \\ Palicot, Preden S Roulleau
}

\section{To cite this version:}

Haïfa Farès, Christian D.C. Glattli, Yves Louët, Christophe Moy, Jacques Palicot, et al.. New Binary Single Side Band CPM. 24th IEEE International Conference on Telecommunications (ICT 2017), May 2017, Limassol, Cyprus. 10.1109/ICT.2017.7998261 . hal-02087957

\section{HAL Id: hal-02087957 https://hal.science/hal-02087957}

Submitted on 2 Apr 2019

HAL is a multi-disciplinary open access archive for the deposit and dissemination of scientific research documents, whether they are published or not. The documents may come from teaching and research institutions in France or abroad, or from public or private research centers.
L'archive ouverte pluridisciplinaire HAL, est destinée au dépôt et à la diffusion de documents scientifiques de niveau recherche, publiés ou non, émanant des établissements d'enseignement et de recherche français ou étrangers, des laboratoires publics ou privés. 


\title{
New Binary Single Side Band CPM
}

\author{
Haïfa Farès ${ }^{\dagger \ddagger}$, Christian Glattli $i^{\ddagger}$, Yves Louët ${ }^{\dagger}$, Christophe Moy $^{\dagger}$, Jacques Palicot ${ }^{\dagger}$, Preden Roulleau ${ }^{\ddagger}$ \\ ${ }^{\dagger}$ IETR - UMR CNRS 6164, CentraleSupélec, avenue de la Boulaie - CS 4760135576 CESSON-SEVIGNE Cedex, France \\ Email: \{haifa.fares, yves.louet, christophe.moy, jacques.palicot\}@ centralesupelec.fr \\ $\ddagger$ CEA Saclay, SPEC, URA 2464, F-91191 Gif-Sur-Yvette, France \{christian.glattli, preden.roulleau\}@cea.fr
}

\begin{abstract}
A new frequency-shift keying continuous phase modulation (CPM) signal, directly inspired from quantum physics, is defined, and its attractive spectral property of single side-band is shown. The principle of the modulator and the demodulator are discussed; and a simplified coherent detector, based on signal correlation using an orthogonal basis, is proposed showing that this new modulation can operate using existing digital communication technology.
\end{abstract}

\section{INTRODUCTION}

Continuous phase modulation (CPM) [1] is known to combine constant signal envelope and good bandwidth efficiency $[2,3]$. For these attractive properties, a significant interest is then addressed to CPM since it was developed in the $80 \mathrm{~s}$ [1], and it has been widely used in digital communication systems, e.g., in satellite communications, deep-space, optical fiber, etc. For instance, CPM has been introduced in the DVB-RCS2 standard [4]. The aim for the use of CPM in these contexts of communication is twofold: first, to exploit their inner immunity against nonlinear distorsions, resulting from the constant envelope; second, to take advantage of their recursive nature allowing their particular use in serially concatenated schemes [5]. Consequently, CPM is classified as power efficient digital modulation, and is therefore often prefered to linear modulations.

Further to the advantages mentioned above, in the present paper, we propose a totally new CPM scheme having the original feature of a single side-band (SSB) spectrum property providing a very compact frequency occupation. This SSB property can be exploited to perform high rate and low frequency bandwidths transmissions without taking care of the image frequency interference, typical of ordinary double side-band signals. The new CPM is hereafter called Single Side-Band Phase Shift Keying (SSB-PSK), which uses a generic phase derivative pulse $d \varphi / d t$ with Lorentzian shape and a $2 \pi$ phase increment. The Lorentzian pulse is a specific shape addressing fundamental quantum physics, in particular, the on-demand injection of a single electron in a quantum conductor. Following a theoretical proposal by Levitov et al. [6], a short voltage pulse $V(t)$ is applied on a contact of the conductor giving a current pulse $I(t)=2 e^{2} V(t) / h_{0}(e$ is the electron charge and $h_{0}$ the Planck constant). Tuning the pulse amplitude and duration such that the net charge $Q=\int I(t) d t=e$, a single electron is injected from the contact to the conductor. However, the voltage pulse perturbs all electrons of the conductor creating unwanted excitations. Levitov et al. in [6] showed that if $V(t)$ has a Lorentzian shape (and only this shape) a pure single electron state is created : a leviton. The experimental demonstration of levitons and their exploitation are given in [7,8] setting the basis for a quantum levitonics. When $V(t)$ is a Lorentzian pulse, the electron energy distribution (equivalently frequency spectrum) becomes SSB. The SSB-PSK is then proposed as first immediate application of a classical levitonics for digital transmissions based on CPM [9].

This paper is organized as follows. In Section II, we first briefly introduce the system model. The fundamental modulation principle is studied in Section III. Then, a low complexity coherent receiver is described in Section IV. Numerical results are reported in Section V. Finally, a conclusion and some perspectives are drawn in Section VI.

\section{System Model}

The CPM signal at carrier frequency $f_{c}$ is defined as

$$
\begin{aligned}
s(t) & =\sqrt{\frac{E_{s}}{T_{s}}} e^{j\left(2 \pi f_{c} t+\varphi(t)\right)} \\
& =\sqrt{\frac{E_{s}}{T_{s}}} e^{\left.j\left(2 \pi f_{c} t+h \sum_{k=-\infty}^{+\infty} a_{k} \varphi_{0}(t-k T)\right)\right),}
\end{aligned}
$$

where $E_{s}$ is the average symbol energy, $T_{s}$ is the duration of symbol $a_{k}, h$ is an integer modulation index ensuring an $2 \pi$ phase increment, $\varphi(t)$ is phase function and $\varphi_{0}(t)$ is a Levitonic phase-shift function, given by

$$
\varphi_{0}(t)=2 \mu \arctan \left(\frac{t}{w}\right) .
$$

The information symbols $a_{k}$ are assumed to be independent and identically distributed and take values in the alphabet $\{0,1\}$, i.e., no antipodal coding is performed in order to preserve the SSB property. $\varphi_{0}(t)$ is the phase response and it is obtained by

$$
\varphi_{0}(t)=\int_{-\infty}^{t} g(\tau) d \tau,
$$

where $g(t)$, the frequency pulse, is a truncated Lorentzian pulse defined as

$$
\begin{aligned}
g(t) & =\frac{d \varphi_{0}(t)}{d t}=\mu \frac{2 w}{t^{2}+w^{2}}, \quad t \in\left[0, L T_{s}\right] \\
\int_{0}^{t} g(\tau) d \tau & =\varphi_{0}\left(L T_{s}\right)=2 \pi, \quad t \geq L T_{s}
\end{aligned}
$$

with $w$ the pulse width, a tuning parameter impacting greatly modulation performance, particularly its spectral efficiency. 


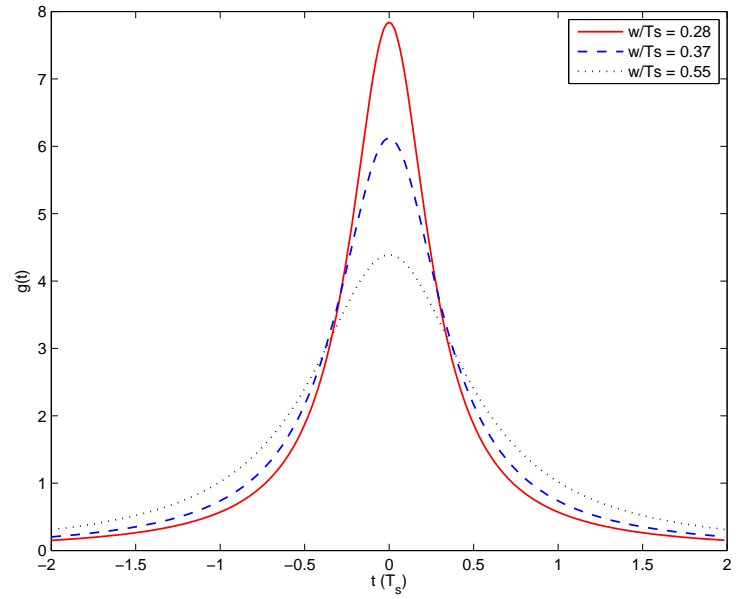

Fig. 1. Length- $4 T_{s}$ frequency pulse: the Lorentzian pulse for different width values, $w / T_{s}=0.28,0.37,0.55$.

Given that the Lorentzian pulse decreases very slowly, $\mu$ is a correcting factor introduced to keep a $2 \pi$ phase increment when the frequency pulse $g(t)$ is truncated to $L>1$ symbol durations. This factor is defined as the ratio between the total phase increment without any truncation and the one obtained after Lorentzian truncation :

$$
\mu=\frac{2 \pi}{\int_{-L T_{s} / 2}^{L T_{s} / 2} \frac{2 w}{t^{2}+w^{2}} d t}=\frac{\pi}{\arctan \left(\frac{L T_{s}}{2 w}\right)} .
$$

For the non-truncated Lorentzian pulse $(L=\infty), \mu=1$.

In figure 1 , we illustrate the Lorentzian pulse, for different width values $w / T_{s}=0.28,0.37,0.55$. As $g(t)$, the frequencyshift function, is partial-response, the SSB-PSK modulation exhibits inter-symbol interference (ISI) which is obviously expected and goes up when $w$ is getting larger. It is therefore imperative to choose a limited width $w$ for Lorentzian pulses to reduce ISI effects on bit error rate (BER) performance.

For the signal given in (1), we perform a phase coding where the $k^{\text {th }}$ bit of duration $T_{s}$ contributes to the total phase $\varphi_{0}(t)$ of the carrier by $2 a_{k} \arctan \left(\frac{t-k T_{s}}{w}\right)$. Equivalently, a Lorentzian pulse with a phase increment equal to $2 \pi$ is used to encode bit 1 and no pulse is associated with bit 0 .

We consider a point-to-point communication system over an additive white Gaussian noise (AWGN) channel. The powerlimited input signal $\mathbf{s}$ is summed to the complex noise $\mathbf{z} \sim$ $\mathcal{C N}\left(0, \sigma_{0}^{2}\right)$ resulting in the output $\mathbf{y}$

$$
\mathbf{y}=\mathbf{s}+\mathbf{z},
$$

with $\sigma_{0}^{2}$ the Gaussian noise variance.

\section{FUNDAMENTAL PRINCIPLE OF SSB-PSK MODULATION}

The spectrum of the complex signal $x(t)=e^{-i \varphi(t)}$ is defined by its Fourier transform $X(f)=\int_{-\infty}^{+\infty} e^{-j \varphi(t)} e^{j 2 \pi f t} d t$. To obtain a single side-band spectrum, this Fourier transform must have a zero value for $f<0$. Therefore, $x(t)$ must have no pole in the lower half-plane and at least one pole in the upper half-plane. Furthermore, $x(t)$ has a constant complex envelope. The simplest solution verifying the conditions mentioned above is then given by

$$
x(t)=e^{-j \varphi(t)}=\frac{t-t_{0}+j w_{0}}{t-t_{0}-j w_{0}} .
$$

For this solution, $x(t)$ has a pole at $t=t_{0}+j w_{0}$, which implies the phase-shift function $\varphi(t)=\varphi_{0}(t)=2 \arctan \left(\frac{t-t_{0}}{w}\right)$, resulting in the Fourier transform $X(f)=e^{-4 \pi w_{0} f}$, for $f>0$ and zero otherwise.

The generalization of this principle is to add poles exclusively in the upper half-plane, which allows $X(f)$ to maintain this original SSB feature. Explicitly, this generalization consists in generating more complex phases, sum of $N$ elementary phases $\varphi_{i}(t)$ of type $\varphi_{0}$, occuring at time $t_{i}$ and of duration $w_{i}\left(w_{i}>0\right)$. The general expression of $x(t)$ is then

$$
x(t)=e^{-j \varphi(t)}=\prod_{i=0}^{N-1} \frac{t-t_{i}+j w_{i}}{t-t_{i}-j w_{i}} .
$$

Likewise, the SSB property is conserved if the phase shift function is generalized to

$$
\varphi(t)=\sum_{i=0}^{N-1} h_{i} \varphi_{i}(t)
$$

where $h_{i}$ are integers of same signs. If $h_{i}>0 \forall i, X(f)$ has zero value for $f<0$; and if $h_{i}<0 \forall i, X(f)$ has zero value for $f>0$. This explains why no antipodal coding is applied in bit-to-symbol conversion before using this principle in phase modulation of information bits.

\section{SSB-PSK DEMODULATION SCHEME}

In this section, we propose a coherent demodulation scheme based on symbol-by-symbol detection, i.e., the receiver makes a decision on one symbol only, based on the observation of one received symbol. The demodulation method relies on the property of orthogonality between SSB-PSK modulated signals with unique pulses of different amplitude levels corresponding to symbol values; for binary modulation, we exploit orthogonality between signals relative to bits 0 and 1 .

\section{A. Orthogonality Property}

Let us denote by $u_{\tilde{h}}(t), \tilde{h}=0,1,2, \ldots, N$ a set of orthogonal functions having the following form

$$
\begin{aligned}
u_{\tilde{h}}(t) & =\frac{1}{\sqrt{2 \pi}} e^{j \tilde{h} \varphi_{0}(t)} \sqrt{\frac{d \varphi_{0}(t)}{d t}} \\
& =\frac{1}{\sqrt{2 \pi}} \sqrt{\frac{(t+j w)^{\tilde{h}}-1}{(t-j w)^{\tilde{h}}}}
\end{aligned}
$$

where $\varphi_{0}(t)$ is a non-truncated Levitonic pulse defined in (2) with $\mu=1$. We can easily verify that $\int_{-\infty}^{+\infty} u_{\tilde{h}}^{*}(t) u_{\tilde{h}^{\prime}}(t) d t=$ 


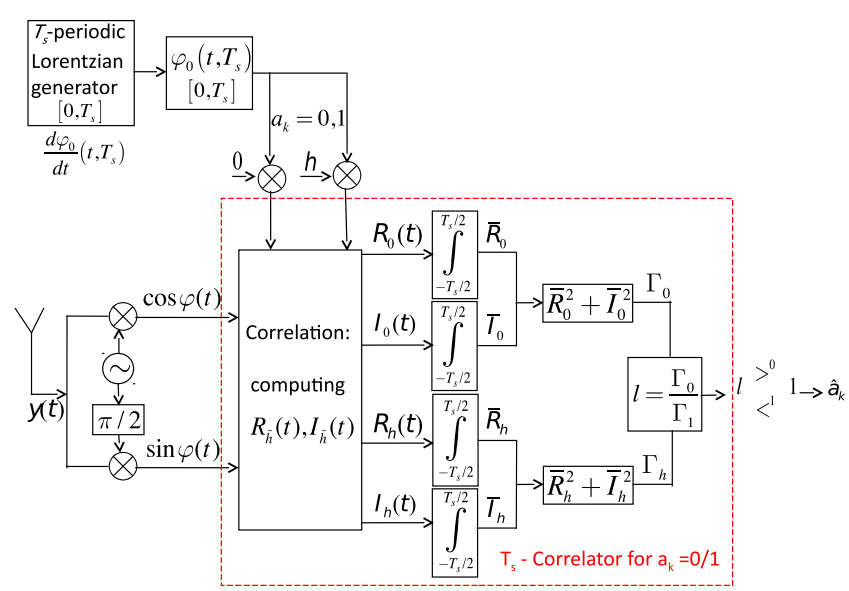

Fig. 2. Block diagram of the correlation-based demodulator.

$\delta_{\tilde{h}, \tilde{h}^{\prime}}$. In order to use this orthogonality property in demodulation process of SSB-PSK modulated signal, we have rather considered the set of signals

$$
s_{\tilde{h}}(t)=e^{j h \varphi_{0}(t)}=\sqrt{\frac{(t+j w)^{\tilde{h}}}{(t-j w)^{\tilde{h}}}} .
$$

The correlation-based separation of signals $s_{\tilde{h}}(t)$ and $s_{\tilde{h}^{\prime}}(t)$ using orthogonality is given then by the following integration formula

$$
\frac{1}{2 \pi} \int_{-\infty}^{+\infty} s_{\tilde{h}}^{*}(t) s_{\tilde{h}^{\prime}}(t) \frac{d \varphi_{0}(t)}{d t} d t=\delta_{\tilde{h}, \tilde{h}^{\prime}}
$$

where $\frac{d \varphi_{0}(t)}{d t}$ is a weighting function in the integration function.

The extension of the definition of the set of orthogonal functions defined over an interval no longer infinite but finite $T_{s}$ is straightforward; and consequently, the orthogonality property is verified thanks to the following integration function

$$
\frac{1}{2 \pi} \int_{-T_{s} / 2}^{+T_{s} / 2} s_{\tilde{h}}^{*}(t) s_{\tilde{h}^{\prime}}(t) \frac{d \varphi_{0}(t)}{d t} d t=\delta_{\tilde{h}, \tilde{h}^{\prime}} .
$$

Moreower, we have to emphasise that the weighting function $\frac{d \varphi_{0}(t)}{d t}$ is a periodic Lorentzian sum and can be precalculated off-line before performing the demodulation process.

\section{B. Binary Correlation-based Demodulator}

For the binary case, the set of orthogonal functions used in the demodulation process consists of two different vectors given by $s_{\tilde{h}}, \tilde{h}=h a_{k}$, where $a_{k}=0,1$. Exploiting orthogonality property, the detection of the transmitted symbol, using the received observation $y(t)$, is based on the computation of the following correlation functions for $\tilde{h}=0, h$

$$
\Gamma_{\tilde{h}}(t)=\left|\int_{t-T_{s} / 2}^{t+T_{s} / 2} y(t) s_{\tilde{h}}^{*}(t) \frac{d \varphi_{0}(t)}{d t} d t\right|,
$$

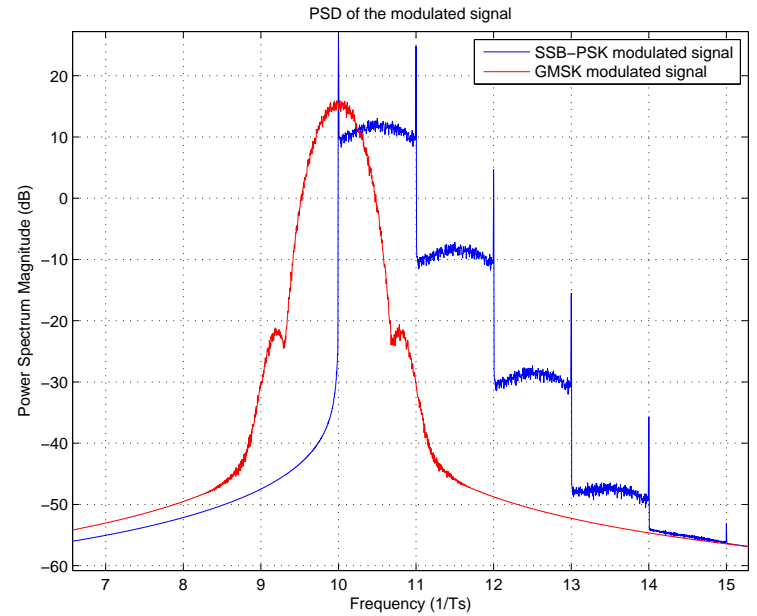

Fig. 3. Comparison of the power spectral densities: in blue for an SSB-PSK signal, in red for a GMSK signal, for the same carrier frequency $f_{c}=10 / T_{s}$.

in order to establish the likelihood ratio test

$$
l=\frac{\Gamma_{0}}{\Gamma_{h}}>_{1}^{0} 1
$$

The demodulator scheme is depicted in figure 2. After carrier demodulation, correlation functions are computed based on noisy inphase and quadrature phase components, $\cos (\varphi(t))$ and $\sin (\varphi(t))$.

\section{NUMERICAL RESULTS AND COMPARISONS}

\section{A. SSB Spectral Property}

The power spectral density (PSD) of the SSB-PSK modulated signal is represented by figure 3 , averaged over 10 spectra corresponding to 10 different 2048 bits length streams each, non truncated Lorentzian pulses of width $w=0.37 T_{s}$ are used, i.e., $L=\infty$ (numerically, we choose a value large enough to guarantee a phase increment equal to $2 \pi$ or $\mu=1$ ). The frequencies are in units of $1 / T_{s}$. The carrier frequency is $f_{c}=10 / T_{s}$. In this example, we choose $h=1$, a positive integer; as a result all the spectral components of the signal are located then in the band greater than $f_{c}$. For comparison, we also present the PSD of the GMSK modulated signal.

The PSD of the SSB-PSK modulated signal is indeed unilateral when comparing it with the GMSK modulated signal which is symmetrical with respect to the carrier frequency $f_{c}$. Almost all of the power is concentrated in a $1 / T_{s}$ frequency band and the power spectrum decreases steeply in steps of 20 $\mathrm{dB}$, spaced by frequential periods of $1 / T_{s}$. This exponential decrease is equal to $e^{-4 \pi w f}$ (see Section III), which is equivalent to $1 / 100$ in linear scale and to $20 \mathrm{~dB}$ in logarithmic scale for $w / T_{s}=0.37$. Consequently, the power exponential decay of the SSB-PSK modulated signal is shown to be proportional to the Lorentzian width $w$. A tradeoff on the value of $w$ has to be found in order to combine the ISI reduction by limiting $w$ ( $c f$. section II) and ensuring a fast power decay outside the useful band by increasing $w$. 


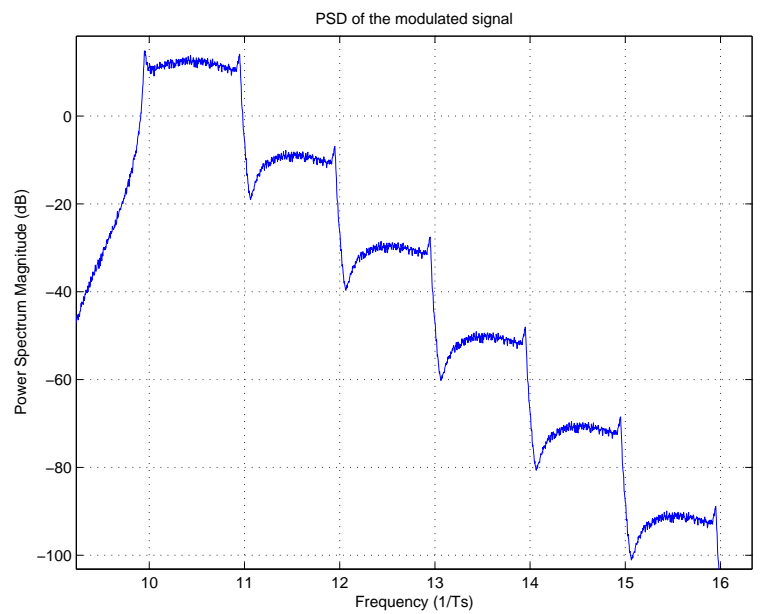

Fig. 4. Power spectral density of an SSB-PSK signal with $95 \%$ of the phase increment of a pure Lorentzian.

Spikes, also called spectral lines, are also observed around the frequencies $f_{c}, f_{c}+1 / T_{s}, f_{c}+2 / T_{s}$, etc. They are due to the value of the phase increment which is equal to $2 \pi$, what is well-known in conventional phase modulation techniques whose phase increment is $2 \pi$ [10]. However, it remains important to emphasize that deviating a little from this critical value will reduce or even eliminate these spectral lines at the cost of a reappearance of very low amplitude components in the lower band. The PSD of the SSB-PSK modulated signal, with a phase increment equal to $95 \%$ of $2 \pi$ is represented on figure 4 . The configuration of the signals considered in figure 3 has been maintained for this illustration. The major difference is the clear reduction of the spectral lines. However, its spectral occupation has slightly increased.

Table I provides the effect of the Lorentzian truncation on the PSD of the SSB-PSK modulated signal. In this table, we present the spectral occupation of the modulated signalfor several values of $L=\infty, 12,4,2$. This spectral occupation, denoted by $B w$, is expressed in terms of $1 / T_{s}$ frequency band occupied by $98 \%$ of the signal power transmitted for $w=$ $0.37 T_{s}$. For comparison, we also give the spectral occupation of a GMSK modulated signal. For instance, for $B T=0.3$, $B w=0.86$. We can clearly note through these results that for non-truncated Lorentzian pulses, the spectral occupation of the SSB-PSK modulation remains close to those reached by the GMSK modulation. However, the more truncated the waveform is, the more spectral occupation increases.

\section{B. Bit Error Rate Performance}

In this section, we investigate the performance of the SSBPSK modulation in terms of BER over an AWGN channel. In particular, we plot in figure 5, BER curves of SSBPSK modulation for several Lorentzian pulse length values $L=100,4,2$, as a function of $E_{b} / N_{0}$ the bit-energy-tonoise ratio. This quantifies the effect of truncation on the performance of the proposed demodulation scheme and thus

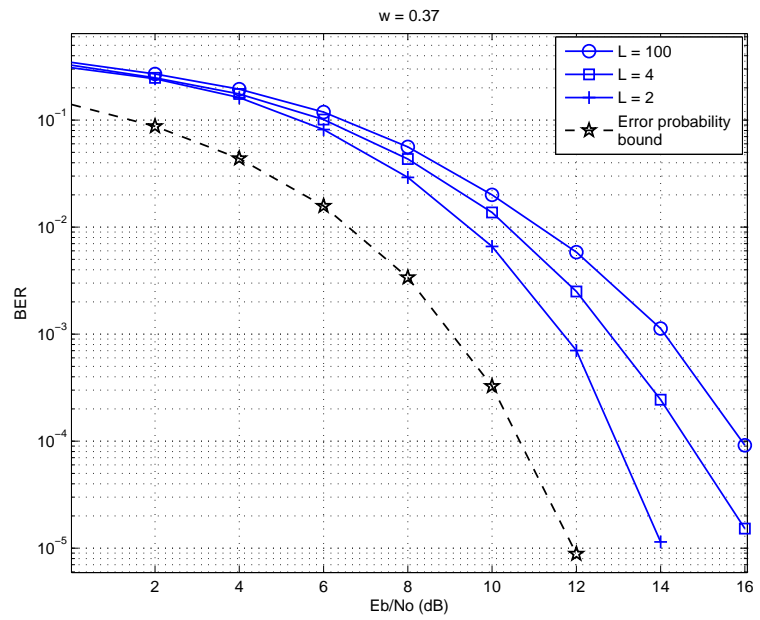

Fig. 5. BER performance for several truncation scenarios $L=100,4,2$.

its immunity against ISI. It is a non-surprizing result that SSB-PSK modulation with the most severe truncation of the Lorentzian pulse is the most efficient, since it is subject to the the lowest ISI level.

Furthermore, a theoretical bound on the bit error probability is given in order to illustrate the potential of this new modulation. This performance bound is described using error events and minimum distance concepts [11]. The normalized squared Euclidian distance of a binary CPM is defined in [1] as

$$
d^{2}=\frac{1}{2 T_{s}} \int\left|s\left(t, \mathbf{a}_{\mathrm{Tx}}\right)-s\left(t, \mathbf{a}_{\mathrm{Rx}}\right)\right|^{2} d t
$$

For an error location arbirarily choosen as $a_{e}$, the error event consists in transmitting the symbol sequence $\mathbf{a}_{\mathrm{Tx}}$ and receiving the symbol sequence $\mathbf{a}_{\mathrm{Rx}}$, defined both as

$$
\begin{aligned}
& \mathbf{a}_{\mathrm{Tx}}=\left(\ldots, a_{e-1}, a_{e}, a_{e+1}\right), \\
& \mathbf{a}_{\mathrm{Rx}}=\left(\ldots, a_{e-1}, \bar{a}_{e}, a_{e+1}\right) .
\end{aligned}
$$

The phases of SSB-PSK modulated signals relative to both $\mathbf{a}_{\mathrm{Tx}}$ and $\mathbf{a}_{\mathrm{Rx}}$ information sequences diverges at position $e$ end converges again due to the $2 \pi$ phase increment. Consequently, the minimum euclidian distance $d_{\min }$ is then relative to a single-symbol error event. The probability of bit error is then bounded as

$$
P_{b} \leq \frac{1}{2} Q\left(\sqrt{d_{\min }^{2} \frac{E_{b}}{N_{0}}}\right)
$$

TABLE I

SPECTRAL OCCUPATION

\begin{tabular}{lllll}
\hline \multicolumn{5}{c}{ SSB-PSK } \\
\hline \hline$L$ & $\infty$ & 12 & 4 & 2 \\
\hline$\mu$ & 1 & 1.04 & 1.13 & 1.29 \\
\hline$B w$ & 1 & 1.08 & 1.26 & 1.53 \\
\hline
\end{tabular}


where $Q(x)=\frac{1}{\sqrt{2 \pi}} \int_{x}^{\infty} e^{-u^{2} / 2} d u$. This performance bound is achieved for the maximum likelihood sequence estimation (MLSE). Assuming $N$ transmitted symbols, the MLSE rule for the estimation of $\mathbf{a}=\left(a_{1}, a_{1}, \ldots, a_{N}\right)$ is given by

$$
\left(\hat{a}_{1}, \hat{a}_{1}, \ldots, \hat{a}_{N}\right)=\underset{\mathbf{a} \in\{0,1\}^{N}}{\operatorname{argmax}}\left|\int_{-N T_{s} / 2}^{N T_{s} / 2} y(t) s^{*}(t, \mathbf{a}) d t\right|^{2} .
$$

The proposed demodulator scheme is a sub-optimal demodulator, since it is based on symbol-by-symbol detection and does not taking into account the memory of the modulated signal. Therefore, a great attention will be addressed to design the optimal demodulation scheme of this new modulation in the future works.

\section{CONCLUSIONS}

We proposed in this paper an original binary CPM scheme directly inspired from quantum physics. We explored its singular property of single-side band spectrum, by comparing its PSD with the one obtained from a conventional GMSK scheme widely used in transmission contexts that can be targeted by this new proposal. Moreover, we developped a simple demodulator. Being a partial response, the demodulator performance has been investigated for several pulse length values in order to evaluate its potentiel. Furthermore, since the phase increment is equal to $2 \pi$, neither the use of Viterbi decoder to perform MLSE detector nor pulse amplitude modulation (PAM) decomposition is straightforward. Therefore, future works will pay more attention to find the optimal demodulator scheme.

\section{ACKNOWLEDGEMENT}

This work is supported by the project ERC Proof of Concept, which is funded by the European research council, under the grant agreement coded: $680875 \mathrm{C}$-Levitonics.

\section{REFERENCES}

[1] J. B. Anderson, T. Aulin and C. E. W. Sundberg, Digital Phase Modulation, New york: Plenum Press, 1986.

[2] C. H. Kuo and K. M. Chugg, "On the bandwidth efficiency of CPM signals," Proc. IEEE Military COmm. Conf. (MILCOM), pp. 218-224, Oct. 2004.

[3] G. E. Corazza, Digital satellite communications, Springer, 2007.

[4] Digital Video Broadcasting (DVB), "Second Generation DVB interactive Sattelite System; Part 2: Lower layers for Satellite standard. DVB Document A155-2," Mar. 2011.

[5] M. Messai, A. Piemontese, G. Colavolpe, K. Amis Cavalec and F. Guilloud, "Binary CPMs with Improved Spectral Efficiency," IEEE communications letters, vol. 20, no 1, pp. 85-8-8, Jan. 2016

[6] L. Levitov, H. Lee and G. Lesovik, "Electron counting statistics and coherent states of electric current," J. Math. Phys., no 37, pp. 4845, Jul. 1996.

[7] J. Dubois, T. Jullien, F. Portier, P. Roche, A. Cavanna, Y. Jin, W. Wegscheider and D. C. Glattli, "Minimal-excitation states for electron quantum optics using levitons," Nature, no 502, pp. 659-663, Oct. 2013.

[8] T. Jullien, P. Roulleau, B. Roche, A. Cavanna, Y. Jin and D. C. Glattli, "Quantum tomography of an electron," Nature, no 514, pp. 603-607, Oct. 2014.

[9] D.C. Glattli and P. Roulleau, patent WO2016124841 A1, 2016.

[10] H. E. Rowe and V. K. Prabhu, "Power spectrum of a digital frequencymodulation signal," The Bell System Technical Journal, vol. 54, no 6, pp 1095-1125, 1975 .

[11] E. Perrins and M. Rice, "Reduced-complexity approach to iterative detection of coded SOQPSK," IEEE Trans. on Comm., vol. 55, no 7, pp. 1354-1362, Jul. 2007. 